\title{
A Proposed Application for Controlling Overweight and Obesity within the Framework of the Internet of Things
}

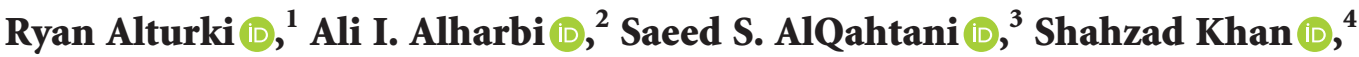 \\ and Vinh Truong Hoang $\mathbb{D}^{5}$ \\ ${ }^{1}$ Department of Information Science, College of Computer and Information Systems, Umm Al-Qura University, \\ Makkah, Saudi Arabia \\ ${ }^{2}$ Department of Information Systems, King Abdulaziz University, Jeddah 215089, Saudi Arabia \\ ${ }^{3}$ Department of Computer Information Systems, Faculty of Computer Science and Information Technology, \\ Imam Abdulrahman Bin Faisal University, Dammam 1982, Saudi Arabia \\ ${ }^{4}$ Computer Science Department, Abdul Wali Khan University Mardan, Mardan 23200, Khyber Pakhtunkhwa, Pakistan \\ ${ }^{5}$ Department of Information Technology Specialization, FPT University, Hoa Lac High Tech Park, Hanoi 10000, Vietnam
}

Correspondence should be addressed to Ryan Alturki; rmturki@uqu.edu.sa

Received 31 August 2021; Revised 31 October 2021; Accepted 12 November 2021; Published 14 December 2021

Academic Editor: omar cheikhrouhou

Copyright (c) 2021 Ryan Alturki et al. This is an open access article distributed under the Creative Commons Attribution License, which permits unrestricted use, distribution, and reproduction in any medium, provided the original work is properly cited.

Currently, overweight and obesity are two problems for global health that affect people's quality of life. The Internet of Things (IoT) has raised the possibility of digital interaction taken to another level. This paper proposes design requirements to develop a mobile application that contributes to the control of overweight or obesity under the IoT approach, focusing on some approaches that currently exist in home-oriented health with the help of existing mobile applications. The idea of this concept is the presence of the environment of various interconnected things or objects with sensors, networking, and processing technologies integrating and working together to provide an environment in which smart services are taken to the end users. The IoT allows monitoring people in real time and analyzing information provided by mobile applications and smart devices (wearables) they have, through techniques of artificial intelligence that allow the identification of variables criticism and the generation of medical recommendations. The medical assistance model will change from the existing hospital-centric model that we commonly know to a totally home-centred health one, in which there will be less work intensity and operational cost. The home health service allows the use of IoT technology, very often called Internet of Healthcare Things (IoHT).

\section{Introduction}

Obesity is a medical condition characterized by the excessive accumulation of body fat. The degree of obesity can be obtained by calculating the Body Index Mass (BMI). This number is obtained when a person's weight in kilograms is divided by the square height of the person in meters. A person whose $\mathrm{BMI}$ is greater than 27 is considered obese [1]. In 2016, more than 1.9 billion adults over the age of 18 in everyone were overweight, and more than 600 million were obese; in addition, 41 million children under the age of five were overweight $[1,2]$. Obese people often get sick from cardiovascular disease, have high blood pressure, diabetes, and difficulty in breathing and walking, and have various other problems. People with obesity often need to be actively monitored and regulated their diet and physical activity [3]. Therefore, it is considered a public health problem that presents great risks in the health sectors in many countries [1].

Nowadays, the development of technology has enabled the creation of and improvement of sensor devices that greatly help in monitoring and controlling health. Smartphones, these days, can be very constructive when it comes to managing healthy lifestyles. It is also easier for doctors to monitor patients with obesity and also to encourage greater the activity of sufferers to help themselves and preserve their 
health. People suffering from obesity often do not know how to deal with this problem and try various treatments and medications that are inefficient and expensive [2]. Currently, the emerging of Internet of Things (IoT) shows an important role in the development of information technologies, networks, and communications. The word "thing" means any physical object that can be different types of devices, vehicles, and infrastructure elements, which are connected to the network, making them unique and cognizable [2]. According to [4], the Internet of Things (IoT) is about connecting devices and objects of everyday life via the Internet and performs some desired tasks. The concept of IoT consists of the interconnection of intelligent objects such as sensors, integrated circuits, and microcontrollers; these embedded systems can simply connect and assemble data. The IoT allows different types of sensors and end devices to be integrated with objects that are connected to the Internet through fixed and wireless networks. Today, with the use of smart mobile devices, more than 14 sensors have been involved to generate raw data like location, motion, and current temperature. Applying the IoT in all types of activities can provide a strong communication platform for all standard types of Internet that enable devices and noninternet-enabled devices through wireless or cable connections to sense, connect, and share data [5]. On the other hand, healthcare has received a lot of attention recently because of the important application area evolving into the field of the IoT, since that it is adapted to include prescriptions, consultation, continuous monitoring system, tracking food intake, and remote capturing. Currently, various medical devices and sensors have been constantly used to monitor and measure patients' medical parameters, such as heart rate, blood glucose level, and body temperature [6]. Measuring and monitoring various parameters of the human body are done by medical sensors, especially in situations where the beneficiary is not near a healthcare facility. This provides real-time insight into the patient's condition, which opens up numerous possibilities in the context of a better understanding of the user's condition and timely responses to health problems [7]. Applications of IoT are rising rapidly due to our needs and demands. Furthermore, the IoT will allow a better quality of life, since it has the ability of collecting and analyzing such data that, together with each other, can become important information and knowledge.

A recent study of [7] cited that there are several types of communication in the concept of IoT: firstly, data exchange between machines/devices (machine-to-machine, M2M), secondly, machine-to-person data exchange (machine-toperson, M2P), and, finally, person-to-person data exchange $(\mathrm{P} 2 \mathrm{P})$. The $\mathrm{M} 2 \mathrm{M}$ communication means that there is a connection between machines and devices without interaction of people. Figure 1 presents the IoT elements.

Many machines and devices can offer efficient healthcare assistances and solutions through applications as well as using mobile devices to deliver healthcare services anytime and anywhere [4]. Much of the current literatures on IoT pay particular attention to the term Internet of Medical Things (IoMT) which is one of the most promising areas in the

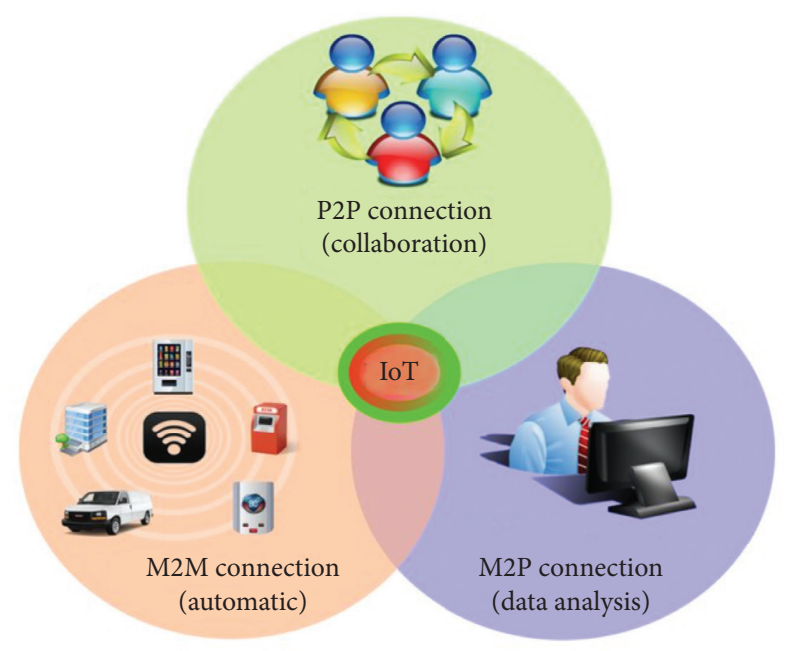

FIgURE 1: The IoT elements that are commonly used to describe the IoT concepts [7].

healthcare industry as it increases efficiency and quality of real-time diagnosis $[8,9]$. The IoMT consists of three main components: the hardware works as a firmware, middleware, and cloud platform works as software [10]. Monitoring and tackling infectious diseases are some examples introduced by the technology of IoMT. Utilizing the biomedical and electronic devices through digital surveillance and tracking and tracing infected individuals becomes the future of digital health and IoMT [9-11]. Figure 2 shows the most common telemedicine services and their workflow in a diagram.

With the advent of smartphones, access to the Internet and social media has largely begun to take place via mobile devices [12]. Due to the popularity of smartphones, new opportunities have emerged to create applications that would serve the purpose of health promotion. Therefore, an architecture for the IoT can help to represent innovation with new digital dimension where a large number of objects acquire greater computing capacity. These innovations have extensive capabilities and awareness of the environment such as energy independence and improving the processing power [13]. Designing an architecture for the IoT includes several aspects such as business models, scalability, and interoperability between heterogeneous devices and their business models processes. Furthermore, the idea of networking, communication, extensibility, and security can involve the design of IoT architecture [14]. Because objects in the IoT transfer geographically and can be authorized to gather data from things and the environment that surround them [13], the recognitions of IoT sensors and the Radio Frequency Identification System (RFID) have allowed machines and computers to understand and recognize the environment through radio waves in real time without the intervention of humans $[14,15]$.

Recently, the design and development of architectures for this purpose have been the focus of many researchers as well as companies that intend to develop devices and services for it. Purpose architecture is the realization of the best possible communication between devices, people, and things 


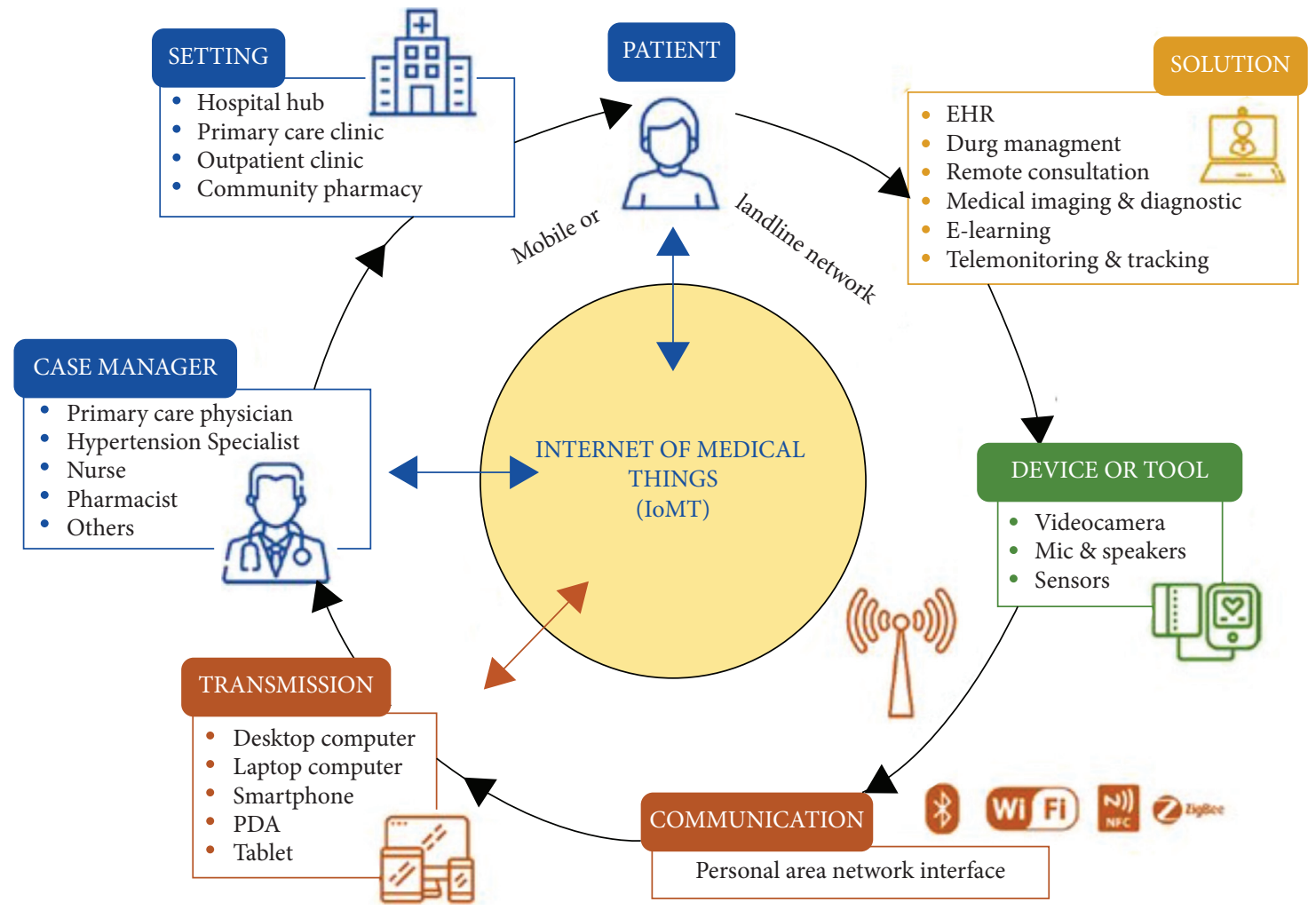

FIgURE 2: A diagram of the most common telemedicine services and their workflow [8].

in the IoT. The role of Service-Oriented Architecture (SOA) is imperative for service providers and users as it also guarantees interoperability between heterogeneous devices in multiple ways [15]. The idea behind SOA is a complex system that has functionalities and capabilities summarized in a common set of services and set of well-defined simple objects or subsystems, where these are maintained individually. As a result, both software and hardware components in the IoT are efficiently reused and then updated. These advantages of SOA are applied for many architectures of wireless sensor networks, because it is intended to provide expansion, scalability, modularity, and mainly interoperability between heterogeneous things $[14,16]$. On the other hand, challenges like the reliability of patient data, security, and privacy need to be addressed in this application domain [17]. In addition, optimizing these challenges can lead to many improvements in the security of medical data, service availability, redundancy, unpredictable performance, legal aspects, and other concerns $[15,18,19]$.

This paper presents the realization of an application for mobile devices based on Android that allows merging with the specified IoT architecture. The application is used to read certain data of the mobile device and send the specified data to something that is a collector, used to collect and process such messages and medical recommendations. That information should be accessible in any way possible connections. Due to the impossibility of creating an application for all mobile platforms, the purpose of this work was developing the application only for the operating system Android. Along with the mobile application, a simple server was created that serves as an intermediary between the application and the simulator that processes the specified data. The server is created due to greater application independence and proper data processing, thus achieving easier changes to specifications of mutual communication. The developed system was intended for the prevention of obesity through an Android mobile application for retrieving data from a web server, analyzing and processing collected data, and providing an accessible user interface to the user.

\section{Background and Related Work}

The evolution of IoT-based devices is beginning to transform the style of lives of many individuals and particularly in the various care activities of the health. In this sense, the use of IoT can be utilized by providing great opportunities for the monitoring, analysis, diagnosis, and recommendations of diseases such as being overweight or obese. For better understanding of the patient's condition, innovated applications like wearable devices and smartphones have facilitated Remote Patient Monitoring (RPM). That is why many researchers focus their research on this topic. The following is a review of the status of the art of works using mobile applications and IoT technologies in healthcare, particularly in relation to being overweight or obese.

The term mHealth is used to represent most of mobile and wireless health technologies. Day by day, there are increasing numbers of applications for smartphones in the market. Furthermore, the evolution of IoMT has provided monitoring applications that led to significant usability 
improvements on many platforms $[11,12,20]$. According to a mobile health report, more than 97,000 were reported to be healthcare applications in the trade of applications on various mobile operating systems [13, 20]. Most of these applications are of general health and physical or fitness type. For example, a research in [1] presented a mobile health application with the purpose of increasing the levels of awareness of parents and children about the risks of obesity and help them in maintaining a lifestyle, balanced and healthy eating. Additionally, the authors in [3] proposed a mobile health architecture aiming at preventing childhood obesity by promoting good health behaviours and prescribing automated manners with a set of mHealth applications that can provide notifications and messages to adults to improve results.

Many mobile applications for a weight loss program with machine-to-machine (M2M) technology have been reviewed by [21], using a special weight scale to upload data to the server. The applications can suggest necessary treatments and facilities that are required for treating the patient and preparing an analysis report for the doctor to make a quick look.

Additionally, in [22], the authors developed a study to evaluate the effectiveness of obesity prevention in children, with a mobile platform system, which is a an agent-based approach that can work together with a wearable device, designed to recommend eating and physical activities and enhance the healthy behaviours to prevent childhood obesity.

Moreover, the authors in [23] developed a device that facilitates measurements of real-time monitoring and stores medical records for multiple patients simultaneously. This IoT device allows obese patients and doctors to acknowledge for any irregular conditions for all measured parameters.

Both papers in $[24,25]$ shared a study of the effectiveness of an experimental intervention based on diet recommendations through a smart app for weight loss in patients over 18 years old, being overweight or obese, concluding that weight loss apps are helpful for people who are ready to control calories. However, for the average overweight patient, it is unlikely that the introduction of a smart phone application produces a significant weight change. Similarly, a study in [26] presented a software architecture that works as a wireless sensor network system for ambulatory monitoring health status. The system consists of several sensor nodes that monitor the body, movement, and activity of the heart, using a network coordinator and personal server. In addition, they used a software architecture that is based on a widely used open-source operating system for networking sensors.

Both papers in $[27,28]$ presented overviews of the existing systems of health monitoring, taking into account the IoT approach, in addition to the recent trends and the development of health monitoring systems in terms of parameters and frameworks of health, wireless communication, and problems of security, as well as identifying limitations and advantages.

The outcomes of IoT and mobile applications on human health are similar to those of $[29,30]$ who presented a system that is able to adequately track the progress of the user's weight loss over time and recommend meals and dietitian program to avoid compromising their health.

Finally, a group of experts have studied and defined the new concept of IoT-learning integrating the IoT technologies. The study of [31] proposed a personalized program training using IoT-learning for weight management and pervasive learning. This proposal provides to users not only personalized information, transmitting data through the network and receiving the information to analyze it, but also data that matches the personalized information. IoTlearning provides users (students) with feedback by collecting information through sensors. The most important goal of this learning is providing personalized training to users with the data obtained through sensor technology in the IoT. This document combines the technology of detection in IoT and learning systems.

Researchers attempted to evaluate the impact of collecting health data through devices and also create challenges regarding how, when, and with whom to share the data, as well as what type of information to share, with what specificity, and for what it will be used $[32,33]$. Data privacy is an ethical issue that raises great concern. In health, the devices will be able to collect information on all kinds of variables, many of them not even thought about years ago, and this will represent new challenges in privacy [34]. Therefore, when thinking about data collection for the clinical area and public health, there is an urgent need to identify and assess risks and thus improve decision-making when adopting and designing a safer and more reliable Internet of Medical Things [35, 36].

Currently, the opportunity to take advantage of Artificial Intelligence (AI) through the IoT is enormous, since statistical and numerical information is obtained from different data such as pressure, heart rate, and movement. It also includes methods for automated perception, computerized learning, understanding, and reasoning, which become commonplace in life [37]. The ultimate objective of AI is to simulate human intelligence; in addition to having great applications in different fields, in which the most important is health, and this field of science owes much of its current development to the results obtained in the process of certain types of medical problems: the diagnosis and treatment of diseases [38, 39].

In the mid-seventies, evidence of efficacy and success in this field were obtained; as of these dates, the scientific communities of medicine and computer science saw their interest growing in this emerging scientific field. AI, in addition to providing wonderful advances, contains a series of advantages and disadvantages [38, 39].

\subsection{Disadvantages}

(i) Because it is software, it requires constant updates (maintenance)

(ii) Time consuming as it needs to be performed with expert systems

(iii) Creates self-sufficient machines and replaces the human race 
(iv) Human feels less important when a machine or a system replaces human's intuition

\subsection{Advantages}

(i) In the workplace, it reduces costs and additional salaries

(ii) Applications are developed performing tasks that humans would not achieve due to the complexity of them

(iii) possible long-term conditions

(iv) reduces time for certain activity

(v) achieves great findings and improvements

It is a type of AI that gives computers the ability of learning, without being explicitly programmed. Machine learning has the ability of capturing information from the environment, solving problems, and adapting to the context; that is, they are intelligent. Within machine learning, algorithms can be classification algorithms, regression algorithms, segmentation algorithms, and association algorithms [38]. Predict one or more discrete variables, based on other attributes in the data set. Among the classification algorithms are the following.

2.3. Decision Trees. A technique allows analyzing sequential decisions based on the use of outcomes and associated probabilities. Decision trees are used to generate expert systems, binary searches, and game trees. All of this have to do with public health, and, in view of this scenario, one could then speak of an intelligent public health, an avant-garde digital context, where this discipline is proactive in the processes of innovation in health and, mainly, in understanding [40].

\section{Theoretical Concepts}

The proposal for the design of a mobile application for the monitoring and control of the overweight or obese addresses a priority issue on a public health problem, such as overweight or obesity, which is intended to be resolved through connectivity, interoperability, and integration of devices in the IoT and incorporating artificial intelligence techniques, applied to healthcare. Therefore, the proposed theoretical concept is divided into three modules and is shown in Figure 3.

3.1. Monitoring. It will make it possible to measure the vital signs of the people who are overweight or obese to identify critical variables (calories burns, sleep, heart rate, Body Mass Index (BMI), number of floors climbs, sleep quality, calories consumed, exercises, and weight control) and use them as parameters to predict problems or pathologies that affect health of a person, through artificial intelligence techniques. Also, in this module, it is intended to identify patterns that improve or affect overweight or obesity in relation to heart rate, food intake, calories burned, water consumption, and

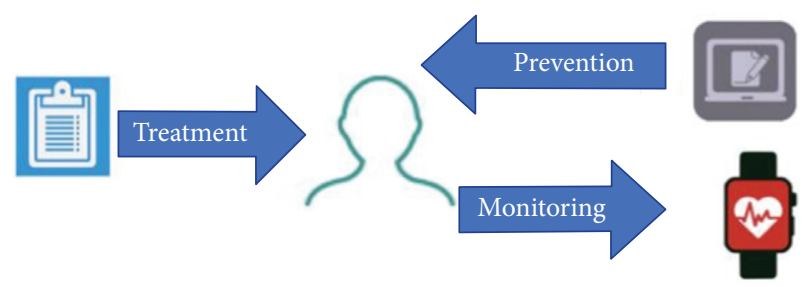

Figure 3: Theoretical concepts.

quality of sleep, among other variables, with the purpose to obtain the necessary information for the generation of recommendations and motivational messages.

3.2. Prevention. This module considers medical recommendations that lead to actions, procedures, and comprehensive interventions that maintain or improve the health of people who are overweight or obese by measuring parameters and the identifying critical variables. Therefore, it is planned to show recommendations in the cases that require it, for example, if the heart rate is higher or lower according to normal values, due to excess or lack of exercise, or if the intake of food decreases the consumption of protein or increases the consumption of fats or carbohydrates.

3.3. Treatment. It consists of supporting users with palliative care needed to treat symptoms and side effects caused by the overweight or obesity; it is also intended to follow up on the recommendations carried out to monitor the evolution and identify which recommendation is the one that provides better results. For the module design, a Unified Modeling Language (UML) class diagram is used, which details the structure of the system that requires a supporting mechanism to get that information from the Application Programming Interface (API) to formulate the advice needed to provide information of interest to users, clinicians, and healthcare providers who integrate with the application without affecting the application's performance.

It is designed in an object-oriented model with 5 main layers:

(1) Presentation layer: this layer maintains direct communication with the user and contains the following classes:

(i) Heart: it is a specific class to keep the information corresponding to heart data, such as number of beats per minute and frequency cardiac

(ii) Sleep: it keeps the necessary elements to monitor the time and depth of sleep

(iii) Device: it maintains the structure for the control of the devices of the username

(iv) Steps: they contain the control of steps and floors raised

(v) Liquid: it maintains the necessary structure for handling the liquid registered, as well as the quantity 


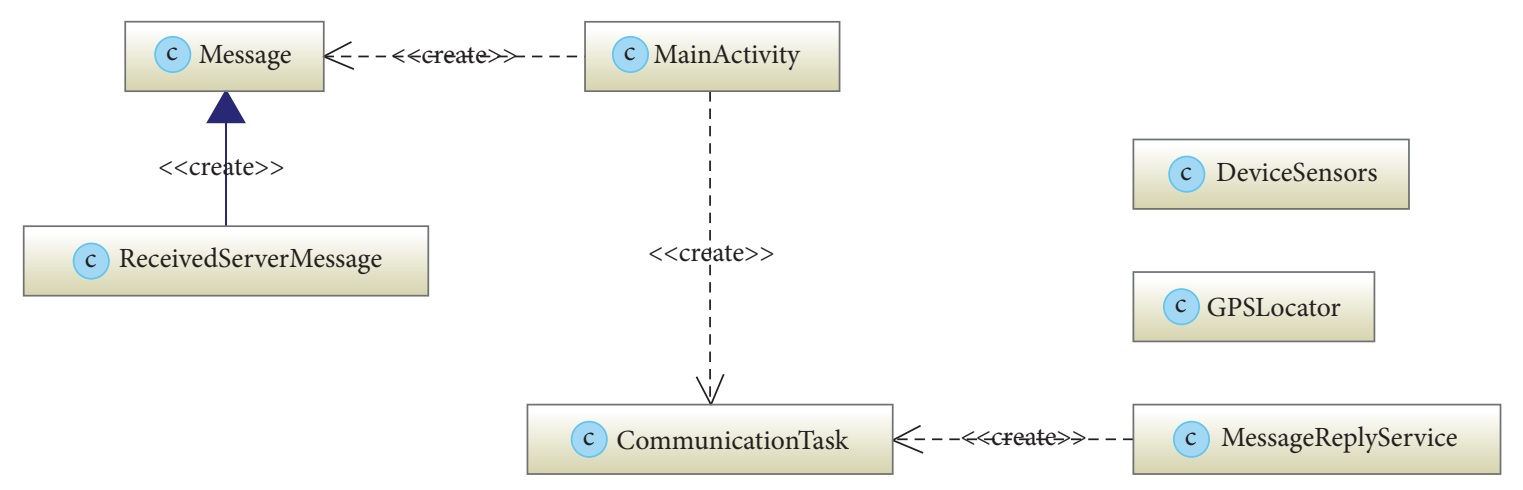

Figure 4: UML basic class diagram.

(v) User: it exemplifies the storage structure of the user

(vi) Exercise: it corresponds to the administration of the exercise carried out

(vii) Intake: it is performed to maintain and control intake information daily

(2) Integration layer: the supplier of the wearable or sensor, the query selector component, and the response trainer are integrated in this layer.

Everybody can collaborate to obtain and access data and then make inquiries from the user and formulate the corresponding responses using the following class:

(i) Engine: in charge of controlling queries, connectors to services and query execution, information processing, and organization, this layer preprocesses the data for easy understanding and consumption.

(3) Services layer: in the IoT, linking, invoking, selecting, and confirming services in IoT are the main responsibilities for this layer. In addition, it contains the following class:

(i) Service: it is responsible for linking and executing medical services corresponding.

(4) Data analytical layer: this layer includes the identification service of critical variables and the medical recommendation system.

(5) Data layer: this layer contains all the beneficial data for the mobile application and the ability of integrating data or events, in real time, and providing critical variables and medical recommendations.

A UML packet diagram is presented in Figure 4.

Likewise, in the UML package diagram, there are three elements that interact with each other: the processing engine and the services in the IoT. A processing engine package, on the contrary, requires a data docking connector to receive stored information and a connection to an information processor that can process and prepare the data received via the connector in the correct format. Ultimately, it can send classification algorithms and recommendations. Additionally, the services in the IoT package contain API that stores the classification and recommendation algorithms to be used in the web application.

\section{Prototype of the Mobile Application}

The proposed mobile application provides a friendly graphical environment that has the objective of contributing to the control of overweight or obesity and the motivation of the user; in addition to displaying statistical data of the information obtained, this flexible design provides you with intake log tools; likewise, it is based on components for the web and mobile devices. Now, it presents some elements that are part of the proposal. Figure 5 presents the UI of the app.

The application consists of an activity and three services. These are classes MainActivity, DeviceSensors, and GPSLocator with MessageReplyService.

The MainActivity serves to display the graphical interface and supports user interaction. Other services are used for background processes that must not be performed on the main user interface (UI) thread. Therefore, services are as follows:

(i) Read and save the values of all sensors, DeviceSensors

(ii) Read and save GPS location values, GPSLocator

(iii) Automatic reply to received messages, MessageReplyService

Services are running when needed. The sensor is constantly running until service for location is interrupted (if it did not give rights to read that data). The service can automatically reply to messages received from users. Once this service is started, it will not stop until the user does so.

Message is a class that represents the basic structure of the message. While ReceivedServerMessage class can extend the database message, it contains additional capabilities needed to process and save received messages. Communication Task is the execution of background task. The purpose of this class is to send a message depending on the method selected (Bluetooth, Wi-Fi, and Internet). Currently, only the ability of sending a message is implemented via Internet.

DeviceSensors is a service that can only be used by the IoT application. Listed service has implemented a SensorEventListener interface that represents the listener for events that cause sensors. SensorManager is used to access the sensors. 


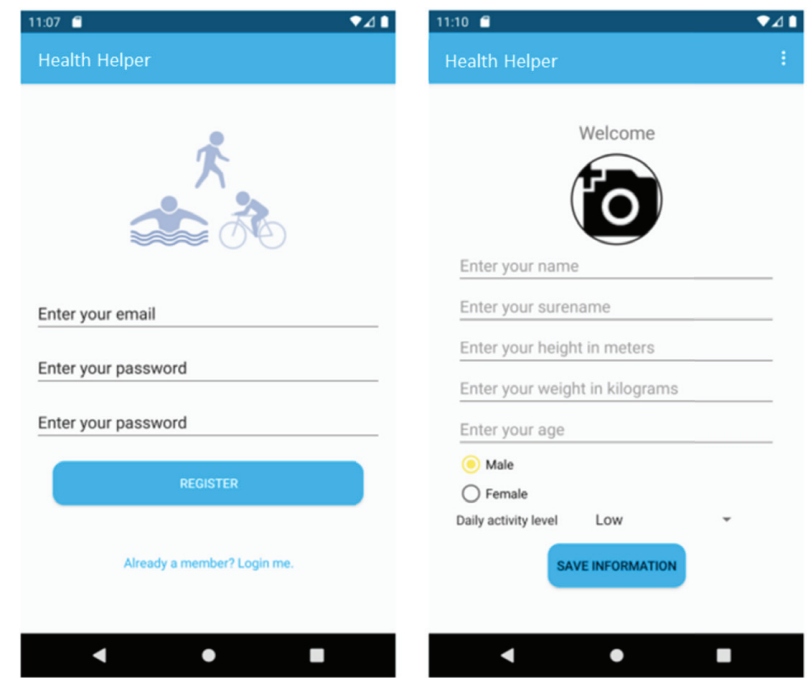

Figure 5: Application's user interface.

TABle 1: Specific sensors that present important data.

\begin{tabular}{ll}
\hline Sensor type & \multicolumn{1}{c}{ Description of sensor values } \\
\hline TYPE_ACCELEROMETER & (i) Measures the force of acceleration \\
TYPE_GRAVITY & (ii) Uses three axes $(x, y$, and $z)$ \\
TYPE_GYROSCOPE & (iii) Unit of measurement: $\mathrm{m} / \mathrm{s}^{2}$ \\
TYPE_LINEAR_ACCELERATION & (i) Measures the room temperature \\
TYPE_AMBIENT_TEMPERATURE & (ii) Unit of measurement: ${ }^{\circ} \mathrm{C}$ \\
TYPE_TEMPERATURE & (i) Measures the brightness of the space \\
TYPE_LIGHT & (ii) Unit of measurement: lx \\
TYPE_MAGNETIC_FIELD & (i) Measures the magnetic field of space \\
TYPE_ORIENTATION & (ii) Uses three axes $(x, y$, and $z)$ \\
TYPE_ROTATION_VECTOR & (iii) Unit of measurement: $\mu \mathrm{T}$ \\
TYPE_PRESSURE & (i) Measures the degree of rotation of the device \\
TYPE_PROXIMITY & through all three axes $(x, y$, and $z)$ \\
TYPE_RELATIVE_HUMIDITY & (i) Measures the air pressure of the room \\
& (ii) Unit of measurement: hPa or mbar \\
& (i) Measures the proximity of an
\end{tabular}

It is necessary to go through all the sensors and subscribe to notifications. The specified sensors of the codes are visible on Table 1.

\section{Conclusion}

The medical area is very extensive, in which to achieve a timely diagnosis it is necessary to verify a set of variables and dependencies, in addition to external factors influencing, such as climate, temperature, and elevation; given the nature of the project, it is difficult to focus directly on a section leaving aside the great variety of aspects that influence overweight or obesity; it was detected that one of the major factors that influence overweight or obesity is the food culture of each region, since it is one of the aspects that are more difficult to change in addition to the fact that many of the products on the market for control or monitoring of overweight or obesity are invasive; that is why the project proposed here was carried out with a noninvasive approach.

Although there is still a long way to go in the aspect of health and its integration with intelligent systems, it must be recognized that the Internet of Things offers great possibilities to provide timely and real-time information on the status of the patient, in search for critical variables such as food control or remote monitoring by a specialist; thanks to this technology and information, lives are currently being saved and in the future real-time diagnoses will be provided, saving patients' time and money.

The IoT paradigm is currently gaining strength in many branches of science and in the management and control of smart homes, people monitoring, among others, since they provide knowledge in real time, but it is important to mention that by itself the IoT only obtains a set of information and knowledge amounts, which must be combined 
with artificial intelligence and data mining techniques to process information and turn it into useful knowledge for users and in this way improve their processes, saving money, energy, or health control, among others.

Additionally, it is known that this project alone is difficult to cover all aspects related to overweight or obesity, but it lays good foundations for future work using mobile application services focused on the Internet of Things, using artificial intelligence techniques and intelligent monitoring for making timely medical recommendations based on the information acquired through wearable devices. Together, the validation by experts in the health area considerably reinforces the project, since it nurtures knowledge to people who do not know issues related to healthcare; it is important and necessary that this type of projects be constantly changed adjusting to new national and international standards that govern the WHO, since it was detected that as times change, the standards are constantly restructured and adjusted according to society.

As technology advances and the IoT gains strength in different branches of science, it is required that more and more experts in different areas of science work together to reach a common goal, since one area of science alone does not cover all the aspects necessary to provide good results; likewise, artificial intelligence algorithms transform the set of raw information amounts into valuable information, seeking or extracting from it the most important and relevant, helping in this decision-making and improving services recommending to users either something they want or things they make their life easier. Even when this research fulfilled its objective and obtained its achievement, it would be very interesting to delve into the problem studied, giving continuity to this work. It is beneficial that more devices and users are included using wearables and linking them to the platform to analyze the information and compare the results obtained.

In addition, it is suggested to study, detail, and incorporate some other aspects that influence overweight or obesity, such as analyzing the location, temperature, atmospheric pressure, and elevation and thus relate factors that directly and indirectly influence overweight or obesity conditions. Additionally, it would be of great benefit to obtain the existing information from a patient monitored in a traditional way and at the same time monitor him/her with the application and wearable devices to compare the results; in this way, the veracity of the results of this proposal would be confirmed. It is of great importance to indicate that all the recommendations made should always be supervised by a specialist, since like many other methods or systems that provide help in controlling overweight or obesity the recommendations made could be counterproductive for the patient's health.

\section{Data Availability}

The data used to support the findings of this study are available from the corresponding author upon request.

\section{Conflicts of Interest}

The authors declare that there are no conflicts of interest regarding the publication of this paper.

\section{References}

[1] M. Alloghani, A. Hussain, D. Al-Jumeily, P. Fergus, O. Abuelma'Atti, and H. Hamden, "A mobile health monitoring application for obesity management and control using the internet-of-things," in Proceedings of the 2016 Sixth International Conference on Digital Information Processing and Communications (ICDIPC), pp. 19-24, IEEE, Beirut, Lebanon, April 2016.

[2] L. Jane, G. Atkinson, V. Jaime, S. Hamilton, G. Waller, and S. Harrison, "Intermittent fasting interventions for the treatment of overweight and obesity in adults aged 18 years and over: a systematic review protocol," JBI Database of Systematic Reviews and Implementation Reports, vol. 13, no. 10, pp. 60-68, 2015.

[3] A. Alnuaimi, S. Rawaf, S. Hassounah, and M. Chehab, "Use of mobile applications in the management of overweight and obesity in primary and secondary care," JRSM open, vol. 10, no. 3, 2019.

[4] R. Alturki, M. J. AlGhamdi, V. Gay, N. Awan, and M. Alshehri, "Privacy, security and usability for IoT-enabled weight loss apps," International Journal of Advanced Computer Science and Applications, vol. 11, 2020.

[5] S. M. Riazul Islam, D. Daehan Kwak, M. Humaun Kabir, M. Hossain, and K. S. Kyung-Sup Kwak, "The internet of things for health care: a comprehensive survey," IEEE access, vol. 3, pp. 678-708, 2015.

[6] L. Farhan, R. Kharel, O. Kaiwartya, M. Quiroz-Castellanos, A. Alissa, and M. Abdulsalam, "A concise review on Internet of Things (IoT)-problems, challenges and opportunities," in Proceedings of the 2018 11th International Symposium on Communication Systems, Networks \& Digital Signal Processing (CSNDSP), pp. 1-6, IEEE, Budapest, Hungary, July 2018.

[7] M. A. Khan, M. T. Quasim, F. Algarni, and A. Alharthi, "Internet of things: on the opportunities, applications and open challenges in Saudi arabia," in Proceedings of the 2019 International Conference on Advances in the Emerging Computing Technologies (AECT), pp. 1-5, IEEE, Munawwarah, Saudi Arabia, February 2020.

[8] S. Omboni, "Connected health: in the right place at the right time," Connected Health, vol. 1, no. 1, pp. 1-6, 2021.

[9] J. Sun, F. Khan, J. Li, M. D. Alshehri, R. Alturki, and M. Wedyan, "Mutual authentication scheme for the deviceto-server communication in the internet of medical things," IEEE Internet of Things Journal, vol. 8, 2021.

[10] E. A. Adeniyi, R. O. Ogundokun, and J. B. Awotunde, "IoMTbased wearable body sensors network healthcare monitoring system," in IoT in Healthcare and Ambient Assisted Living, pp. 103-121, Springer, Singapore, 2021.

[11] J. N. S. Rubí and P. R. D. L. Gondim, "Interoperable internet of medical things platform for e-health applications," International Journal of Distributed Sensor Networks, vol. 16, no. 1, 2020.

[12] P. C. Santana-Mancilla, L. E. Anido-Rifón, J. ContrerasCastillo, and R. Buenrostro-Mariscal, "Heuristic evaluation of an IoMT system for remote health monitoring in senior care," International Journal of Environmental Research and Public Health, vol. 17, no. 5, Article ID 1586, 2020.

[13] I. Machorro-Cano, U. Ramos-Deonati, G. Alor-Hernández et al., "An IoT-based architecture to develop a healthcare smart platform," in Proceedings of the International Conference on Technologies and Innovation, pp. 133-145, Springer, Cham, Switzerland, October 2017.

[14] S. K. Mishra and A. Sarkar, "Service-oriented architecture for internet of things: a semantic approach," Journal of King Saud University-Computer and Information Sciences, vol. 24, 2021. 
[15] J. Huang, X. Wu, W. Huang, X. Wu, and S. Wang, "Internet of things in health management systems: a review," International Journal of Communication Systems, vol. 34, no. 4, Article ID e4683, 2021.

[16] H. Alqarni, W. Alnahari, and M. T. Quasim, "Internet of things (IoT) security requirements: issues related to sensors," in Proceedings of the 2021 National Computing Colleges Conference (NCCC), pp. 1-6, IEEE, Taif, Saudi Arabia, March 2021.

[17] Quasim and M. Tabrez, Challenges and Applications of Internet of Things (IoT) in Saudi Arabia. No. 5316, Easy Chair, London, UK, 2021.

[18] K. Shafique, B. A. Khawaja, F. Sabir, S. Qazi, and M. Mustaqim, "Internet of things (IoT) for next-generation smart systems: a review of current challenges, future trends and prospects for emerging 5G-IoT scenarios," IEEE Access, vol. 8, pp. 23022-23040, 2020.

[19] S. Nasiri, F. Sadoughi, M. Tadayon, and A. Dehnad, "Security requirements of internet of things-based healthcare system: a survey study," Acta Informatica Medica, vol. 27, no. 4, p. 253, 2019.

[20] O. AlShorman, B. AlShorman, M. Al-khassaweneh, and F. Alkahtani, "A review of internet of medical things (IoMT) based remote health monitoring through wearable sensors: a case study for diabetic patients," Indonesian Journal of Electrical Engineering and Computer Science, vol. 20, no. 1, pp. 414-422, 2020.

[21] S. Garg and P. Pundir, "MOFit: a framework to reduce obesity using machine learning and IoT," 2021, https://arxiv.org/abs/ 2108.08868 .

[22] J. T. de Souza, G. A. L. de Campos, C. Rocha et al., "An agent program in an IoT system to recommend activities to minimize childhood obesity problems," in Proceedings of the 35th Annual ACM Symposium on Applied Computing, pp. 654-661, Brno, Czech Republic, March 2020.

[23] D. Gupta, A. Parikh, and R. Swarnalatha, "Integrated healthcare monitoring device for obese adults using internet of things (IoT)," International Journal of Electrical and Computer Engineering, vol. 10, no. 2, 2020.

[24] R. Alturki and V. Gay, "The development of an Arabic weightloss app Akser Waznk: qualitative results," JMIR formative research, vol. 3, no. 1, Article ID e11785, 2019.

[25] J. Mae, E. Oey, and F. S. Kristiady, "IoT based body weight tracking system for obese adults in Indonesia using realtime database," Earth and Environmental Science, vol. 426, no. No. 1, Article ID 012143, 2020.

[26] U. B. Mahadevaswamy, "Wireless wearable smart healthcare monitoring using android," International Journal of Computer Network and Information Security, vol. 12, no. 2, p. 12, 2018.

[27] J. B. Awotunde, R. G. Jimoh, S. O. Folorunso, E. A. Adeniyi, K. M. Abiodun, and O. O. Banjo, "Privacy and security concerns in IoT-based healthcare systems," in The Fusion of Internet of Things, Artificial Intelligence, and Cloud Computing in Health Care, pp. 105-134, Springer, Cham, Switzerland, 2021.

[28] S. Nazir, Y. Ali, N. Ullah, and I. García-Magariño, "Internet of things for healthcare using effects of mobile computing: a systematic literature review," Wireless Communications and Mobile Computing, vol. 2019, Article ID 5931315, 20 pages, 2019.

[29] S. M. Sefa-Yeboah, K. Osei Annor, V. J. Koomson, F. K. Saalia, M. SteinerAsiedu, and G. A. Mills, "Development of a mobile application platform for SelfManagement of obesity using artificial intelligence techniques," International Journal of Telemedicine and Applications, vol. 2021, Article ID 6624057, 16 pages, 2021.

[30] R. Alturki, M. J. AlGhamdi, V. Gay, N. Awan, M. Kundi, and M. Alshehri, "Analysis of an eHealth app: privacy, security and usability," International Journal of Advanced Computer Science and Applications, vol. 11, no. 4, pp. 209-214, 2020.

[31] I. Machorro-Cano, G. Alor-Hernández, M. A. ParedesValverde, U. Ramos-Deonati, J. L. Sánchez-Cervantes, and L. Rodríguez-Mazahua, "PISIoT: a machine learning and IoTbased smart health platform for overweight and obesity control," Applied Sciences, vol. 9, no. 15, Article ID 3037, 2019.

[32] A. I. Alharbi, V. Gay, M. J. AlGhamdi, R. Alturki, and H. J. Alyamani, "Towards an application helping to minimize medication error rate," Mobile Information Systems, vol. 2021, Article ID 9221005, 7 pages, 2021.

[33] H. Ahmadi, G. Arji, L. Shahmoradi, R. Safdari, M. Nilashi, and M. Alizadeh, "The application of internet of things in healthcare: a systematic literature review and classification," Universal Access in the Information Society, vol. 18, no. 4, pp. 837-869, 2019.

[34] S. L. Holzmann and C. Holzapfel, "A scientific overview of smartphone applications and electronic devices for weight management in adults," Journal of Personalized Medicine, vol. 9, no. 2, p. 31, 2019.

[35] F. H. Alqahtani, "The application of the Internet of Things in healthcare," Int. J. Comput. Appl, vol. 180, no. 18, pp. 19-23, 2018.

[36] R. Alturki, "Research onion for smart IoT-enabled mobile applications," Scientific Programming, vol. 2021, Article ID 4270998, 9 pages, 2021.

[37] A. A. Qaffas, R. Hoque, and N. Almazmomi, "The internet of things and big data analytics for chronic disease monitoring in Saudi Arabia," Telemedicine and e-Health, vol. 27, no. 1, pp. 74-81, 2021.

[38] A. Chattopadhyay, S. Mishra, and A. González-Briones, "Integration of machine learning and IoT in healthcare domain," in Hybrid Artificial Intelligence and IoT in Healthcare, pp. 223-244, Springer, Singapore, 2021.

[39] M. Ibrahim, M. Wedyan, R. Alturki, M. A. Khan, and A. AlJumaily, "Augmentation in healthcare: augmented biosignal using deep learning and tensor representation," Journal of Healthcare Engineering, vol. 2021, Article ID 6624764, 9 pages, 2021.

[40] A. Alsswey, H. AlSamarraie, and B. Bervell, "mHealth technology utilization in the Arab world: a systematic review of systems, usage, and challenges," Health Technology, vol. 11, pp. 1-13, 2021. 